\title{
Spherical collapse model in time varying vacuum cosmologies
}

\author{
Spyros Basilakos, ${ }^{1}$ Manolis Plionis, ${ }^{2}$ and Joan Solà ${ }^{3}$ \\ ${ }^{1}$ Academy of Athens, Research Center for Astronomy and Applied Mathematics, Soranou Efesiou 4, 11527, Athens, Greece \\ ${ }^{2}$ Institute of Astronomy \& Astrophysics, National Observatory of Athens, Thessio 11810, Athens, Greece \\ and Instituto Nacional de Astrofísica, Óptica y Electrónica, 72000 Puebla, Mexico \\ ${ }^{3}$ High Energy Physics Group, Department Estructura i Constituents de la Matèria, Universitat de Barcelona, \\ Diagonal 647, 08028 Barcelona, Catalonia, Spain and Institut de Ciències del Cosmos, UB, Barcelona, Spain
} (Received 27 May 2010; published 12 October 2010)

\begin{abstract}
We investigate the virialization of cosmic structures in the framework of flat Friedmann-LemaitreRobertson-Walker cosmological models, in which the vacuum energy density evolves with time. In particular, our analysis focuses on the study of spherical matter perturbations, as they decouple from the background expansion, "turn around," and finally collapse. We generalize the spherical collapse model in the case when the vacuum energy is a running function of the Hubble rate, $\Lambda=\Lambda(H)$. A particularly wellmotivated model of this type is the so-called quantum field vacuum, in which $\Lambda(H)$ is a quadratic function, $\Lambda(H)=n_{0}+n_{2} H^{2}$, with $n_{0} \neq 0$. This model was previously studied by our team using the latest high quality cosmological data to constrain its free parameters, as well as the predicted cluster formation rate. It turns out that the corresponding Hubble expansion history resembles that of the traditional $\Lambda$ CDM cosmology. We use this $\Lambda(t) \mathrm{CDM}$ framework to illustrate the fact that the properties of the spherical collapse model (virial density, collapse factor, etc.) depend on the choice of the considered vacuum energy (homogeneous or clustered). In particular, if the distribution of the vacuum energy is clustered, then, under specific conditions, we can produce more concentrated structures with respect to the homogeneous vacuum energy case.
\end{abstract}

DOI: 10.1103/PhysRevD.82.083512

PACS numbers: 98.80. $-\mathrm{k}, 95.35 .+\mathrm{d}, 95.36 .+\mathrm{x}$

\section{INTRODUCTION}

Several cosmological observations (supernovae type Ia, $\mathrm{CMB}$, galaxy clustering, etc.) have converged to a paradigm of a cosmic expansion history that involves a spatially flat geometry and a recently initiated accelerated expansion of the Universe (see [1-6] and references therein). From a theoretical point of view, an easy way to explain such expansion is to consider an additional energy component, usually called dark energy (DE) with negative pressure, that dominates the Universe at late times. The simplest DE candidate corresponds to the cosmological constant (see [7-9] for reviews). An elegant model that accurately fits the current observational data is the spatially flat concordance $\Lambda \mathrm{CDM}$ model, which includes cold dark matter (DM) and a cosmological constant, $\Lambda$. However, the $\Lambda$ model suffers, among others [10], from two fundamental problems: (a) The "old" cosmological constant problem (or fine-tuning problem) i.e., the fact that the observed value of the vacuum energy density $\left(\rho_{\Lambda}=\Lambda c^{2} / 8 \pi G \simeq\right.$ $10^{-47} \mathrm{GeV}^{4}$ ) is many orders of magnitude below the value found using quantum field theory [7], and (b) the coincidence problem [11] i.e., the fact that the matter energy density and the vacuum energy density are of the same order (just prior to the present epoch), despite the fact that the former is a rapidly decreasing function of time while the latter is stationary.

Many authors have attempted to solve the above problems (see $[8,9,12]$ and references therein), the key approach being the replacement of the constant vacuum energy either with a time evolving DE (quintessence and the like [8]), or alternatively with a time varying vacuum energy density, $\rho_{\Lambda}(t)$ [13-18]. In the original scalar field models [19] and later in the quintessence context, one can ad hoc introduce an adjusting or tracker scalar field $\phi[20]$ [different from the usual standard model Higgs field], rolling down the potential energy $V(\phi)$, which could mimic the DE [8,9,21-24]. However, it was realized that the idea of a scalar field rolling down some suitable potential does not really solve the problem because $\phi$ has to be some high energy field of a grand unified theory (GUT), and this leads to an unnaturally small value of its mass, namely, one which is beyond all conceivable standards in particle physics. As an example, utilizing the simplest form for the potential of the scalar field, $V(\phi)=m_{\phi}^{2} \phi^{2} / 2$, the present value of the associated vacuum energy density is $\rho_{\Lambda}=$ $\langle V(\phi)\rangle \sim 10^{-11} \mathrm{eV}^{4}$, so for $\langle\phi\rangle$ of order of a typical GUT scale near the Planck mass, $M_{P} \sim 10^{19} \mathrm{GeV}$, the corresponding mass of $\phi$ is expected in the ballpark of $m_{\phi} \sim H_{0} \sim 10^{-33} \mathrm{eV}$.

Notice that the presence of such a tiny mass scale in scalar field models of DE is generally expected also on the basis of structure formation arguments $[25,26]$, namely, from the fact that the DE perturbations seem to play an insignificant role in structure formation for scales well below the sound horizon. The main reason for this homogeneity of the DE is the flatness of the potential, which is necessary to produce a cosmic acceleration. Being the mass associated with the scalar field fluctuation, proportional to the second derivative 
of the potential itself, it follows that $m_{\phi}$ will be very small and one expects that the magnitude of DE fluctuations induced by $\phi$ should be appreciable only on length scales of the order of the horizon. Thus, equating the spatial scale of these fluctuations to the Compton wavelength of $\phi$ (hence to the inverse of its mass) it follows once more that $m_{\phi} \lesssim$ $H_{0} \sim 10^{-33} \mathrm{eV}$. All in all, it appears that the problem that one is creating along with the introduction of $\phi$ is far more worrisome than the problem one is intending to solve, for one is postulating a mass scale which is 30 orders of magnitude below the mass scale associated with the value of the vacuum energy density $\left(m_{\Lambda} \equiv \rho_{\Lambda}^{1 / 4} \sim 2.3 \times 10^{-3} \mathrm{eV}\right)$.

The analysis of the recent cosmological observations indicates that the $\mathrm{DE}$ equation of state (EOS) parameter $w\left(\equiv P_{\mathrm{DE}} / \rho_{\mathrm{DE}}\right)$ is close to -1 to within $\pm 10 \%$, if it is assumed to be constant [1-6], while it is much more poorly constrained if it varies with time [3]. More than two decades ago, Ozer and Taha [27] proposed a time varying $\Lambda$ as a possible candidate to solve the two fundamental cosmological puzzles; see also [28,29] and references therein. In this cosmological paradigm, the dark energy EOS parameter $w$ is strictly equal to -1 , but the vacuum energy density (or $\Lambda$ ) does evolve with time. Of course, the weak point in this approach is the unknown functional form of $\Lambda(t)$, which is however also the case for the vast majority of the DE models. Indeed, in the aforementioned $\Lambda(t)$ models, the evolution law is purely phenomenological [30], without a concrete link to fundamental physics, such as the quantum field theory (QFT) in curved space-time [31]. As emphasized in [32], a completely consistent formulation along these lines should eventually be developed, and such investigations could well be at the heart of one of the most important endeavors of theoretical cosmology in the years to come. Therefore, the study of cosmic perturbations in these models is very important $[33,34]$ as they might reveal surprises not foreseen in the context of the scalar field models. The new effects may have impact both on the cosmological and the astrophysical domains. While we recently analyzed the potential implications on the former [35], here we focus on the latter domain.

A pioneering QFT fundamental approach to variable $\Lambda$ models was actually proposed long ago within the context of the renormalization group (hereafter RG) in [36,37]. Later on, the RG-running framework was further explored from different points of view in [13-15], and a more systematic presentation from the viewpoint of QFT in curved space-time by employing the standard perturbation RG techniques of particle physics appeared in [14,16]. Subsequent elaborations, and comparison with the observational tests, confirmed the phenomenological viability of this approach [38-44].

In the class of RG models we shall focus on, the vacuum energy density is expected to vary with time according to the law [14,38-41]: $\Lambda=n_{0}+n_{2} H^{2}$ (hereafter called the $\Lambda_{\mathrm{RG}}$ model or quantum field vacuum model). As already mentioned, in Ref. [35] we have investigated thoroughly the global dynamics of this cosmological model [together with various alternative $\Lambda(t)$ models], in light of the most recent cosmological data. However, a serious issue here is how the main features of the largest collapsed cosmic structures, i.e., galaxy clusters, are affected by a running vacuum energy density. We have argued above that this problem can be addressed in scalar field models of the DE, but only at the expense of admitting extremely tiny mass scales which are uncommon in particle physics. In this paper, we wish to further explore the alternative option in which the DE component is a time evolving cosmological term $\Lambda=\Lambda(t)$, and in this way to assess if the clustering properties of the vacuum energy can shed some light on the fundamental issue of structure formation.

The so-called spherical collapse model [45], which has a long history in cosmology, is a simple but still a fundamental tool used to describe the growth of bound systems in the Universe via gravitation instability [46]. In the last decade many authors have studied the small scale dynamics using this model and found that the main features of the cosmic structures (collapse factor, virial density, etc.) can potentially be affected by the presence of dark energy $[25,26,47-66]$. The aim of the present work is to generalize the spherical collapse model within the variable $\Lambda_{\mathrm{RG}}$ cosmological model, in order to understand nonlinear structure formation in such cosmologies and investigate the differences with respect to the expectations of the concordance $\Lambda \mathrm{CDM}$ cosmology.

The structure of the paper is as follows. The basic theoretical elements of the problem are presented in Sec. II, where we introduce [for a spatially flat FriedmannLemaitre-Robertson-Walker (FLRW) geometry] the basic cosmological equations. In Sec. III we generalize the virial theorem in the case of the QFT $\Lambda(t)$ cosmological model. Section IV outlines the theoretical analysis of the spherical collapse model in which the vacuum energy density varies with the cosmic time, and in Sec. V we compare the corresponding theoretical predictions of the different models and present a first attempt to use observational data to constrain the different models. We draw our conclusions in Sec. VI. In Appendix A we remind the reader of some basic elements of the concordance $\Lambda \mathrm{CDM}$ model in order to appreciate the fact that the $\Lambda_{\mathrm{RG}}$ cosmology is an interesting extension of the standard model. Finally, in Appendix B we provide some basic mathematical formulas, while in Appendix $\mathrm{C}$ we provide accurate fitting formulas for a few important parameters, i.e., the density contrast at the turnaround redshift and at the epoch of virialization, which do not have a simple fully analytical form.

\section{COSMOLOGY WITH A TIME-DEPENDENT VACUUM}

The cosmological constant contribution to the curvature of space-time is represented by the $\Lambda g_{\mu \nu}$ term on the 
left-hand side of Einstein's equations. The latter can be absorbed on the right-hand side of these equations

$$
R_{\mu \nu}-\frac{1}{2} g_{\mu \nu} R=8 \pi G \tilde{T}_{\mu \nu},
$$

where the modified $\tilde{T}_{\mu \nu}$ is given by $\tilde{T}_{\mu \nu} \equiv T_{\mu \nu}+g_{\mu \nu} \rho_{\Lambda}$. Here $\rho_{\Lambda}=\Lambda /(8 \pi G)$ is the vacuum energy density associated with the presence of $\Lambda$ (with pressure $P_{\Lambda}=-\rho_{\Lambda}$ ), and $T_{\mu \nu}$ is the ordinary energy-momentum tensor of isotropic matter and radiation. Modeling the expanding Universe as a perfect fluid with velocity 4 -vector field $U_{\mu}$, we have $T_{\mu \nu}=-P_{m} g_{\mu \nu}+\left(\rho_{m}+P_{m}\right) U_{\mu} U_{\nu}$, where $\rho_{m}$ is the proper isotropic pressure of matter radiation and $P_{m}$ is the corresponding pressure. Clearly the modified $\tilde{T}_{\mu \nu}$ defined above takes the same form as $T_{\mu \nu}$ with $\tilde{\rho}=\rho_{m}+$ $\rho_{\Lambda}$ and $\tilde{p}=P_{m}+P_{\Lambda}=P_{m}-\rho_{\Lambda}$, that is $\tilde{T}_{\mu \nu}=$ $-\tilde{p} g_{\mu \nu}+(\tilde{\rho}+\tilde{p}) U_{\mu} U_{\nu}$. Explicitly,

$$
\tilde{T}_{\mu \nu}=\left(\rho_{\Lambda}-P_{m}\right) g_{\mu \nu}+\left(\rho_{m}+P_{m}\right) U_{\mu} U_{\nu} .
$$

With this generalized energy-momentum tensor, and in the spatially flat FLRW metric $d s^{2}=d t^{2}-a^{2}(t) d \vec{x}^{2}$, the gravitational field equations boil down to Friedmann's equation

$$
H^{2} \equiv\left(\frac{\dot{a}}{a}\right)^{2}=\frac{8 \pi G}{3} \tilde{\rho}=\frac{8 \pi G}{3}\left(\rho_{m}+\rho_{\Lambda}\right),
$$

and the dynamical field equation for the scale factor:

$$
\frac{\ddot{a}}{a}=-\frac{4 \pi G}{3}(\tilde{\rho}+3 \tilde{p})=-\frac{4 \pi G}{3}\left(\rho_{m}+3 P_{m}-2 \rho_{\Lambda}\right) .
$$

Let us next contemplate the possibility that $\rho_{\Lambda}=\rho_{\Lambda}(t)$ is a function of the cosmic time. This is allowed by the cosmological principle embodied in the FLRW metric. The Bianchi identities (which ensure the covariance of the theory) then imply $\nabla^{\mu} \tilde{T}_{\mu \nu}=0$. With the help of the FLRW metric, the previous identity amounts to the following generalized local conservation law:

$$
\dot{\rho}_{m}+\dot{\rho}_{\Lambda}+3 H\left(\rho_{m}+P_{m}+\rho_{\Lambda}+P_{\Lambda}\right)=0,
$$

where the overdot denotes derivative with respect to the cosmic time. The above equation can also be derived by combining Eqs. (3) and (4) since it is a first integral of the equations of motion. Notice that we keep $G$ strictly constant, and therefore the assumption $\dot{\rho}_{\Lambda} \neq 0$ necessarily requires some energy exchange between matter and vacuum, e.g. through vacuum decay into matter, or vice versa. ${ }^{1}$ This possibility was first considered by Bronstein in a rather early paper [68].

\footnotetext{
${ }^{1}$ There exists also the possibility that the vacuum is time evolving and nevertheless noninteracting with matter. In this case, however, either the DE has another component apart from $\Lambda$ - see the $\Lambda$ XCDM framework of [17]—or Newton's coupling is also time varying, i.e. $\dot{G} \neq 0[34,39,41,67]$.
}

Let us remark that the EOS of the vacuum energy density maintains the usual form $P_{\Lambda}(t)=-\rho_{\Lambda}(t)=$ $-\Lambda(t) / 8 \pi G$ despite the fact that $\Lambda(t)$ evolves with time. In the matter dominated epoch $\left(P_{m}=0\right)$, Eq. (5) leads to the following energy exchanging balance between matter and vacuum:

$$
\dot{\rho}_{m}+3 H \rho_{m}=-\dot{\rho}_{\Lambda} .
$$

The second Friedmann's equation (4) is formally unchanged by the presence of a time-variable vacuum energy, and in the matter epoch simply reads

$$
\frac{\ddot{a}}{a}=-\frac{4 \pi G}{3}\left(\rho_{m}-2 \rho_{\Lambda}\right) .
$$

At this point it is worth noticing that the effect of having a variable cosmological term $\rho_{\Lambda}=\rho_{\Lambda}(t)$ cannot, in general, be described by the simple parametrizations usually employed for the effective EOS $w=w(t)$ of the DE, in which $w$ depends on two parameters $\left(w_{0}, w_{1}\right)$ that can be constrained using observations $[21,69]$. The effective EOS of a variable vacuum model is in general more complicated. This is shown in detail, with specific examples, in Ref. [40]. In particular, the vacuum models that we are going to consider cannot be described with these simple parametrizations. Therefore, the variable vacuum models must be studied on their own and constitute an independent class of DE models.

Combining Eqs. (3) and (6), we find

$$
\dot{H}+\frac{3}{2} H^{2}=4 \pi G \rho_{\Lambda}=\frac{\Lambda}{2} .
$$

If the vacuum term is negligible, $\Lambda \rightarrow 0$, then the solution of Eq. (8) reduces to that of the Einstein-de Sitter model, $H(t)=2 / 3 t$, as it should. Similarly, the traditional $\Lambda=$ const cosmology (or $\Lambda \mathrm{CDM}$ concordance model) also follows directly by integrating Eq. (8) (see Appendix A). Finally, this same equation is also valid for $\Lambda=\Lambda(t)$ when matter and vacuum become coupled, and in this case a supplementary equation for the time evolution of $\Lambda$ is needed in order to unveil the dynamics of this model. It is interesting to mention here that the link in Eq. (6) between $\dot{\rho}_{m}$ and $\dot{\Lambda}$ is important because interactions between DM and DE could provide possible solutions to the cosmological coincidence problem. This is the reason for which several papers have been published recently in this area $[17,18,70]$, proposing either that the $\mathrm{DE}$ has various interacting components or that the $\mathrm{DE}$ and $\mathrm{DM}$ could be coupled. In the following sections, we briefly introduce (for more details see [35]) the cosmological models used in this study.

\section{A. The $\Lambda(t)$ model from quantum field theory}

In this scenario, we use the vacuum solution proposed in [14,38-41] using the RG in quantum field theory (hereafter $\Lambda_{\mathrm{RG}}$ model). The model is characterized by the evolution 
law $\Lambda(H)=n_{0}+n_{2} H^{2}$, in which both coefficients $n_{0}$ and $n_{2}$ are nonvanishing. An equivalent and more convenient parametrization is

$$
\Lambda(H)=\Lambda_{0}+3 \gamma\left(H^{2}-H_{0}^{2}\right),
$$

where $\gamma$ is a constant, which can be positive or negative but small: $|\gamma| \leq 1 / 12 \pi$ [35]. It determines the amount of running of $\Lambda(t)$. Note that the form (9) is convenient because the vacuum energy density is normalized to the present value $\Lambda_{0} \equiv \Lambda\left(H_{0}\right)=3 \Omega_{\Lambda} H_{0}^{2}$. As we will see, all the equations and conditions derived below are equivalent to those of the concordance $\Lambda \mathrm{CDM}$ cosmology (cf. Appendix A) and reduce exactly to them for $\gamma=0$.

From Eqs. (8) and (9) we can easily derive the corresponding Hubble flow as a function of time [35]:

$$
H(t)=H_{0} \sqrt{\frac{\Omega_{\Lambda}-\gamma}{1-\gamma}} \operatorname{coth}\left[\frac{3}{2} H_{0} \sqrt{\left(\Omega_{\Lambda}-\gamma\right)(1-\gamma) t}\right] .
$$

The scale factor of the Universe, $a(t)$, evolves as

$$
a(t)=a_{1} \sinh ^{2 / 3(1-\gamma)}\left[\frac{3}{2} H_{0} \sqrt{\left(\Omega_{\Lambda}-\gamma\right)(1-\gamma)} t\right]
$$

where

$$
a_{1}=\left(\frac{\Omega_{m}}{\Omega_{\Lambda}-\gamma}\right)^{1 / 3(1-\gamma)}
$$

Inverting Eq. (11) we determine the cosmic time as a function of the scale factor:

$$
t(a)=\frac{2}{3 \tilde{\Omega}_{\Lambda}^{1 / 2}(1-\gamma) H_{0}} \sinh ^{-1}\left(\sqrt{\frac{\tilde{\Omega}_{\Lambda}}{\tilde{\Omega}_{m}}} a^{3(1-\gamma) / 2}\right),
$$

where we have introduced

$$
\tilde{\Omega}_{m}=\frac{\Omega_{m}}{1-\gamma}, \quad \tilde{\Omega}_{\Lambda}=\frac{\Omega_{\Lambda}-\gamma}{1-\gamma} .
$$

Let us define the Hubble rate normalized to its current value, $E(a)=H(a) / H_{0}$. Eliminating the cosmic time from Eqs. (10) and (11), one can prove that

$$
\begin{aligned}
E^{2}(a) & =\frac{\Omega_{\Lambda}-\gamma}{1-\gamma}+\frac{\Omega_{m}}{1-\gamma} a^{-3(1-\gamma)} \\
& =1+\Omega_{m} \frac{a^{-3(1-\gamma)}-1}{1-\gamma},
\end{aligned}
$$

or

$$
E^{2}(a)=\tilde{\Omega}_{\Lambda}+\tilde{\Omega}_{m} a^{-3(1-\gamma)} .
$$

Notice that both sets of cosmological parameters satisfy the standard cosmic sum rule:

$$
\tilde{\Omega}_{m}+\tilde{\Omega}_{\Lambda}=1=\Omega_{m}+\Omega_{\Lambda} .
$$

Let us also consider the evolution of the matter and vacuum energy densities in this model. Starting from the conservation law [see Eq. (6)] and utilizing Eqs. (3) and (9), we arrive at a simple differential equation for the matter density,

$$
\dot{\rho}_{m}+3 H \rho_{m}=3 \gamma H \rho_{m} .
$$

Using $\dot{\rho}_{m}=a H d \rho_{m} / d a$, we can trivially integrate the previous equation in the scale factor variable, yielding

$$
\rho_{m}(a)=\rho_{m 0} a^{-3(1-\gamma)},
$$

where $\rho_{m 0}$ denotes the matter density at the present time $(a=1)$, and therefore $\Omega_{m}=\rho_{m 0} / \rho_{c 0}$, where $\rho_{c 0}=$ $3 H_{0}^{2} /(8 \pi G)$ is the current critical density. The previous equation can also be rewritten by considering the instantaneous critical density $\rho_{c}(a)$ when the scale factor is $a=a(t)$, i.e. $\rho_{c}(a) \equiv 3 H^{2}(a) /(8 \pi G)$. In fact, defining $\Omega_{m}(a) \equiv \rho_{m}(a) / \rho_{c}(a)$ it is easy to see, with the help of (19) and the definition of $E(a)$, that

$$
\Omega_{m}(a)=\frac{\Omega_{m} a^{-3(1-\gamma)}}{E^{2}(a)} .
$$

Finally, upon inserting (19) in (6) and integrating once more in the scale factor variable, we arrive at the explicit expression for the evolution of the vacuum energy density:

$$
\Lambda(a)=\Lambda_{0}+8 \pi G \frac{\gamma \rho_{m 0}}{1-\gamma}\left[a^{-3(1-\gamma)}-1\right] .
$$

It is important to emphasize from Eq. (19) that the matter density no longer evolves as $\rho_{m}(a)=\rho_{m 0} a^{-3}$, as it presents a correction in the exponent. This is due to the fact that matter is exchanging energy with the vacuum and this is reflected in the corresponding behavior of $\Lambda(a)$ in Eq. (21). As expected, for $\gamma \rightarrow 0\left(\tilde{\Omega}_{m} \sim \Omega_{m}, \tilde{\Omega}_{\Lambda} \sim \Omega_{\Lambda}\right)$ all the above equations reduce to the canonical form within the concordance model (for more details, see Appendix A). Clearly, the usual $\Lambda$ cosmology is a particular solution of the $\Lambda_{\mathrm{RG}}$ model with $\gamma$ strictly equal to 0 . Throughout the rest of the paper we shall employ the statistical results for this model obtained in [35] from a simultaneous fit to the latest SNIa + BAO + CMB data, namely $\Omega_{m}=0.28_{-0.01}^{+0.02}$ (or $\tilde{\Omega}_{m} \simeq 0.281$ ) and $\gamma=0.002 \pm 0.001$.

\section{GENERALIZATION OF THE VIRIAL THEOREM}

Recall that for systems with potential energy of the form $U \propto R^{n}$ the contribution to the virial condition is $2 T-$ $n U=0$, where $T$ is the kinetic energy. For gravity, $n=-1$, whereas for constant vacuum energy $n=2$. Indeed, from Newton's limit of Einstein's equations in the presence of a $\Lambda$ term, i.e. Poisson equation $\nabla^{2} \Phi=$ $4 \pi G\left(\rho_{m}-2 \rho_{\Lambda}\right)$, it follows that the potential associated with a constant $\Lambda$ is $\Phi_{\Lambda}=-(1 / 6) \Lambda R^{2}$. Thus, the nominal virial condition when there is a constant vacuum energy 
reads $2 T+U_{G}-2 U_{\Lambda}=0$, where $U_{G}$ and $U_{\Lambda}$ are the potential energy and the vacuum potential energy, respectively, for an isolated system. However, for nonconstant $\Lambda$ this recipe may not hold anymore. Therefore, the link between the time-dependent vacuum and matter is expected to modify nontrivially the previous form of the virial theorem.

In this section, we generalize the virial theorem by taking into account the presence of the coupling between the vacuum and the matter energy densities, which leads to a variable $\Lambda(t)$. In particular, we have to modify the wellknown Layzer-Irvine equation, which describes the flow to virialization [46]. As we have already stated in Sec. II A [see Eq. (6)], the matter is exchanging energy with the vacuum and this is reflected in the corresponding matter continuity equation (18). Of course, the continuity equation holds for the total cosmic fluid. Since we are interested in cosmic structures which live in high density environments, it is fair to consider that the corresponding inhomogeneous density $n_{m}$ is far from the background homogeneous density, $\rho_{m}$.

The total velocity of the fluid elements, $\mathbf{v}$, is given by $\nabla \cdot\left(n_{m} \mathbf{v}\right)=3 H n_{m}+\nabla \cdot\left(n_{m} \mathbf{u}\right)$, where $\mathbf{u}$ is the peculiar velocity. Note that $\mathbf{v}$ is the sum of the global Hubble expansion plus the line of sight peculiar velocity, $\mathbf{u}$. The continuity equation for $n_{m}$, in the presence of a running vacuum energy $(\gamma \neq 0)$, can now be modified as follows:

$$
\dot{n}_{m}+3 H n_{m}+\nabla \cdot\left(n_{m} \mathbf{u}\right)=3 \gamma H n_{m} .
$$

Notice that if the background would not be expanding $(H=0)$, then Eq. (22) boils down to the standard nonrelativistic continuity equation for a fluid of particles flowing with a velocity distribution $\mathbf{u}$. Based on a Newtonian formulation, the acceleration due to gravity in comoving coordinates is

$$
\frac{d(a \mathbf{u})}{d t}=-a \nabla \Phi,
$$

where $\Phi$ is the gravitational potential. Multiplying Eq. (23) by the quantity $a n_{m} \mathbf{u}$ and using the continuity equation (22) together with the overall Poisson equation [46] $\nabla^{2} \Phi=4 \pi G\left(n_{m}-2 \rho_{\Lambda}\right)$, we can integrate over the volume in order to define the generalized Layzer-Irvine equation. After some calculations we arrive at

$$
\frac{d\left(a^{2} T\right)}{d t}-3 \gamma a^{2} H T=-a^{2}\left(\frac{d \mathcal{U}}{d t}+H \mathcal{U}\right)+6 \gamma a^{2} H \mathcal{U}
$$

where $\mathcal{U}=U_{G}-2 U_{\Lambda}$,

$$
\begin{gathered}
T=\frac{1}{2} \int u^{2} n_{m} d V, \\
U_{G}=-\frac{1}{2} G \iint \frac{n_{m}(x) n_{m}\left(x^{\prime}\right)}{\left|x-x^{\prime}\right|} d V d V^{\prime},
\end{gathered}
$$

and

$$
U_{\Lambda}=-\frac{1}{2} G \iint \frac{n_{m}(x) \rho_{\Lambda}\left(x^{\prime}\right)}{\left|x-x^{\prime}\right|} d V d V^{\prime} .
$$

For a spherical mass fluctuation $M=4 \pi n_{m} R^{3} / 3$, one can show that the above potential energies become

$$
U_{G}=-\frac{16 \pi^{2} G}{3} \int_{0}^{R} x^{4} n_{m}^{2}(x) d x=-\frac{3 G M^{2}}{5 R}
$$

and

$$
U_{\Lambda}=-\frac{16 \pi^{2} G}{3} \int_{0}^{R} x^{4} \rho_{\Lambda}(x) n_{m}(x) d x=-\frac{\Lambda M}{10} R^{2},
$$

where the last equality holds for a homogeneous vacuum energy $\Lambda=\Lambda(a)$ (see [56,58] and Sec. V).

Now, for a system that reaches the equilibrium (virial regime, and $\dot{U}=\dot{T}=0$ ) we derive the condition

$$
(2-3 \gamma) T+(1-6 \gamma)\left(U_{G}-2 U_{\Lambda}\right)=0
$$

or

$$
\frac{U_{G}-2 U_{\Lambda}}{T}=-2 \frac{1-3 \gamma / 2}{1-6 \gamma}=-2-9 \gamma+\mathcal{O}\left(\gamma^{2}\right),
$$

where the last equality is valid for small values of $\gamma$. For $\gamma$ strictly equal to zero we obviously recover the nominal virial theorem in the concordance cosmology $\left(\frac{U_{G}-2 U_{\Lambda}}{T}=-2\right)$ as it should. In the case of $\gamma \simeq 1 / 12 \pi$ [35], which corresponds to receiving quantum effects on the $\Lambda$ running from fields just at the Planck scale $[14,38]$, the above ratio in the $\Lambda(t)$ cosmology deviates by $\sim 12 \%$ with respect to that of the $\Lambda$ cosmology. In practice, however, $\gamma \simeq 1 / 12 \pi$ is excluded by the latest fit to the combined data, which yields a quite smaller value $\gamma \simeq 0.002$ [35]. This value corresponds to quantum effects from GUT fields just 1 order of magnitude below $M_{P}$. In this case, the deviation from the nominal virial condition is only $\sim 1 \%$.

\section{THE SPHERICAL COLLAPSE MODEL}

Despite its simplicity, the spherical collapse model [45] is still a powerful tool for understanding how a small spherical patch of homogeneous overdensity forms a bound system via gravitation instability; for a review, see e.g. [71]. Technically speaking, the basic dynamical cosmological equation (7) is valid both for the entire Universe and also for a homogeneous spherical perturbation. In the last case, we just replace the scale factor $a(t)$ with the radius $R(t)$, and we obtain the so-called Raychaudhuri equation:

$$
\frac{\ddot{R}}{R}=-\frac{4 \pi G}{3}\left[\rho_{m s}-2 \rho_{\Lambda s}\right],
$$

where $\rho_{m s}$ and $\rho_{\Lambda s}$ refer to the corresponding values of the matter and vacuum energy densities in the spherical patch susceptible of ulterior collapse. 
In order to address the issue of how the time varying vacuum energy itself affects the gravitationally bound systems (clusters of galaxies), one has to deal in general with the following three distinct scenarios, which have been considered within different approaches in the literature [25,26,47-66]: (i) the situation in which the vacuum energy remains homogeneous and only the corresponding matter virializes; (ii) the case with clustered vacuum energy, but now assuming that only the matter virializes; and, finally, (iii) the case with clustered vacuum energy, considering that the whole system virializes (both matter and vacuum components). In this paper, we are going to focus on scenarios (i) and (iii) within the framework of time varying vacuum energy density.

From now on, we will call $a_{t}$ the value of the scale factor of the Universe where the spherical overdensity reaches its maximum expansion (i.e. when $\dot{R}=0$ ) and $a_{c}$ the scale factor when the sphere virializes, implying that a cosmic structure has formed. Similarly, $R_{t}$ and $R_{c}$ stand for the corresponding radii of the spherical overdensity, the former being the turnaround (or "top hat") value at the point of maximum size, and the latter refers to the eventual situation when the sphere has already collapsed and virialized. Note that due to the coupling between the time-dependent vacuum and the matter component one would expect that the matter density in the spherical region should obey the same power law as the background matter $\rho_{m}(a) \propto$ $a^{-3(1-\gamma)}$ [see Eq. (19)]. Thus, $\rho_{m s} \propto R^{-3(1-\gamma)}$ denotes the matter density in the spherical patch. Analogously, the vacuum energy density in the spherical region, $\rho_{\Lambda s}$ will take the form (21) with appropriate replacement of the scale factor with $R$; see further below.

From the theoretical point of view, the time needed for a spherical shell to recollapse is twice the turnaround time, $t_{f} \simeq 2 t_{t}$, which implies that [see Eq. (13)]

$$
\sinh ^{-1}\left[\sqrt{\tilde{r}_{0} a_{c}^{3(1-\gamma)}}\right] \simeq 2 \sinh ^{-1}\left[\sqrt{\tilde{r}_{0} a_{t}^{3(1-\gamma)}}\right],
$$

where $\tilde{r}_{0}=\tilde{\Omega}_{\Lambda} / \tilde{\Omega}_{m}$.

In the main panel of Fig. 1, we present the turnaround redshift, $z_{t}=\left(1-a_{t}\right) / a_{t}$, as a function of the virial redshift $z_{c}=\left(1-a_{c}\right) / a_{c}$ for our $\Lambda_{\mathrm{RG}}$ model with $\gamma=0.002$ (continuous line), $\gamma=1 / 12 \pi$ and $\gamma=-1 / 12 \pi$ (long and short dashed lines, respectively). The concordance $\Lambda$ cosmology is indicated by empty points. The relative fractional differences between the $\Lambda_{\mathrm{RG}}$ models and the concordance $\Lambda$ model are extremely small, a fact which can be appreciated in the inset panel of Fig. 1.

As it is evident there is a tight correlation between the two redshifts, which for our $\Lambda_{\mathrm{RG}}$ model $(\gamma=0.002)$ it is given by

$$
z_{t} \simeq 1.532 z_{c}+0.751 .
$$

As an example, assuming that galaxy clusters have virialized at the present time, $z_{c} \simeq 0$, the turnaround epoch takes place at $z_{t} \simeq 0.75$ (or $a_{t} \sim 0.57$ ). On the other hand,

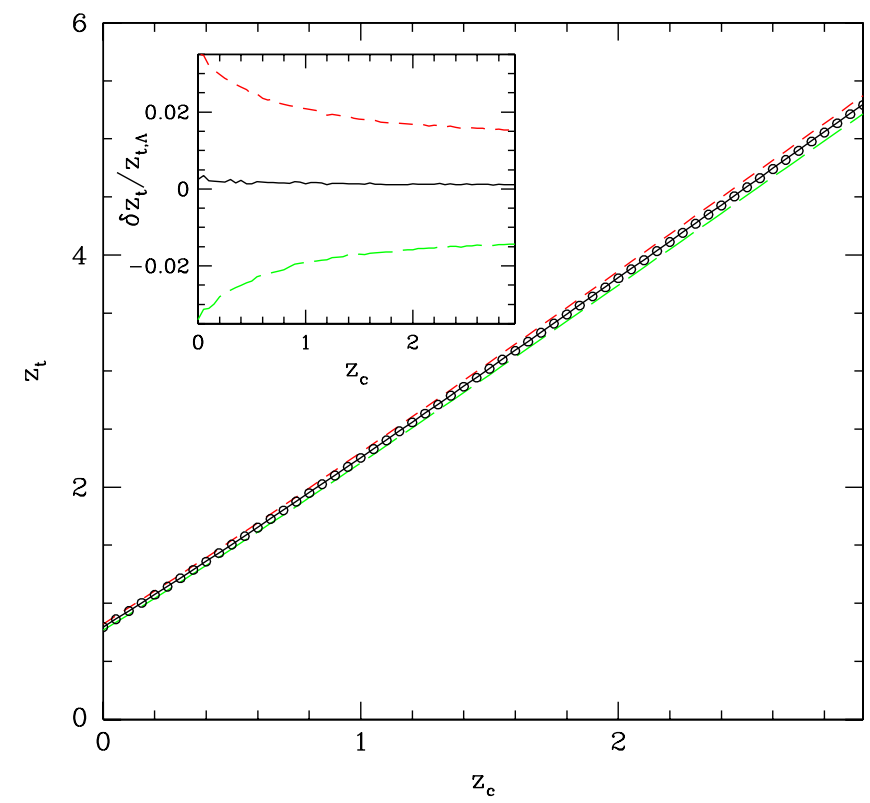

FIG. 1 (color online). The turnaround redshift as a function of the virial redshift; see Eq. (34). The solid, long dashed, and short dashed lines represent the $\Lambda_{\mathrm{RG}}$ model, for $\gamma=0.002,1 / 12 \pi$, and $-1 / 12 \pi$, respectively. The solid points correspond to the concordance $\Lambda \mathrm{CDM}$ cosmology. Inset: The relative fractional difference between the three $\Lambda_{\mathrm{RG}}$ models and the concordance model.

considering that clusters have formed prior to the epoch of $z_{c} \sim 1.6\left(a_{c} \sim 0.38\right)$, in which the most distant cluster has been found [72], the turnaround epoch is not really affected by the vacuum energy component, i.e. $z_{t} \sim 3.2$ (or $a_{t} \sim$ 0.24). This is to be expected, due to the fact that at large redshifts matter dominates the Hubble expansion. It is worth noting that, at high redshifts, the ratio between the scale factors approaches the Einstein-de Sitter $\left(\tilde{\Omega}_{m}=\right.$ $\left.\Omega_{m}=1\right)$ value $a_{c} / a_{t}=\left(1+z_{t}\right) /\left(1+z_{c}\right)=2^{2 / 3}$.

Performing the convenient transformations into dimensionless variables

$$
x=\frac{a}{a_{t}} \quad \text { and } \quad y=\frac{R}{R_{t}},
$$

the evolution of the scale factor of the background and of the perturbation [see Eqs. (3), (16), and (32)] are governed, respectively, by the following two equations:

$$
\left(\frac{\dot{x}}{x}\right)^{2}=H_{t}^{2} \Omega_{m, t}\left[x^{-3(1-\gamma)}+\frac{\rho_{\Lambda}}{\rho_{m, t}}\right]
$$

and

$$
\frac{\ddot{y}}{y}=-\frac{H_{t}^{2} \Omega_{m, t}}{2}\left[\frac{\zeta}{y^{3(1-\gamma)}}-2 \frac{\rho_{\Lambda s}}{\rho_{m, t}}\right],
$$

where $H_{t}^{2} \Omega_{m, t}=\frac{8 \pi G}{3} \rho_{m, t}, \Omega_{m, t}$ is the matter density parameter at the turnaround epoch [see Eq. (20)]. ${ }^{2}$ Note that

\footnotetext{
${ }^{2}$ We set $\Omega_{m, t} \equiv \Omega_{m}\left(a_{t}\right)$, with $\Omega_{m}(a)$ given by Eq. (20)
} 
we have parametrized the matter density in the spherical region at the turnaround time $\rho_{m s, t}=\zeta \rho_{m, t}$, with respect to the background matter density at the same epoch $\rho_{m, t}$. The parameter $\zeta$ is referred to as the density contrast at the turnaround point. In replacing the quantity $\rho_{m s}$ in Eq. (32) we have utilized the following relation:

$$
\rho_{m s}=\rho_{m s, t}\left(\frac{R}{R_{t}}\right)^{-3(1-\gamma)}=\frac{\zeta \rho_{m, t}}{y^{3(1-\gamma)}} .
$$

Therefore, using Eqs. (14) and (21), we find that

$$
\frac{\rho_{\Lambda}}{\rho_{\Lambda, t}}=I(x)=\frac{1+\gamma \tilde{r}_{0} a_{t}^{-3(1-\gamma)} x^{-3(1-\gamma)}}{1+\gamma \tilde{r}_{0} a_{t}^{-3(1-\gamma)}},
$$

with $\rho_{\Lambda, t}$ being the vacuum energy density at the turnaround epoch and $\Omega_{\Lambda, t}=1-\Omega_{m, t}$ is the effective vacuum density parameter at the same time [for definition see Eq. (20)]. Inserting now Eq. (39) into Eq. (36), we finally obtain

$$
\dot{x}^{2}=H_{t}^{2} \Omega_{m, t}\left[x^{-1+3 \gamma}+r x^{2} I(x)\right],
$$

where

$$
r=\frac{\rho_{\Lambda, t}}{\rho_{m, t}}=\frac{\Omega_{\Lambda}}{\Omega_{m}} a_{t}^{3(1-\gamma)}+\frac{\gamma}{1-\gamma}\left[1-a_{t}^{3(1-\gamma)}\right]
$$

[to derive the latter equality we have used Eqs. (17), (19), and (21)].

Of course for the Einstein-de Sitter case $\left(\tilde{\Omega}_{m}=\Omega_{m}=\right.$ 1 and $\gamma=0$ ) the solution of the system formed by Eqs. (36) and (37) reduces to the well-known value of the density contrast at the turnaround point, $\zeta=\left(\frac{3 \pi}{4}\right)^{2}$, as it should. Within this framework, utilizing both the virial theorem [see Eq. (30)] and the energy conservation $\left(T_{c}+\right.$ $U_{G, c}+U_{\Lambda, c}=U_{G, t}+U_{\Lambda, t}$ ) at the collapse time and at the turnaround epoch, respectively, we derive the following useful relation:

$$
q_{1} U_{G, c}+q_{2} U_{\Lambda, c}=U_{G, t}+U_{\Lambda, t},
$$

where

$$
q_{1}(\gamma)=\frac{1+3 \gamma}{2-3 \gamma}, \quad q_{2}(\gamma)=\frac{4-15 \gamma}{2-3 \gamma} .
$$

\section{A. Homogeneous vacuum energy}

In this section, we consider that the vacuum energy component of the scale of galaxy clusters can be treated as being homogeneous: $\rho_{\Lambda s}(a)=\rho_{\Lambda}(a)=\Lambda(a) / 8 \pi G$ (hereafter the $\Lambda_{\mathrm{RGH}}$ model). Therefore, inserting Eq. (39) into Eq. (37), we obtain

$$
\ddot{y}=-\frac{H_{t}^{2} \Omega_{m, t}}{2}\left[\frac{\zeta}{y^{2-3 \gamma}}-2 r y I(x)\right] .
$$

The numerical solution for the $\zeta$ parameter is provided by integrating the main system of differential equations [Eqs. (40) and (44)], using the boundary conditions $(d y / d x)=0$ and $y=1$ at $x=1$. Following the methodology of $[48,65]$ we provide in Appendix C a reasonably accurate fitting formula for $\zeta$, as a function of the main cosmological parameters.

Using now, the combined equation (42) for the potential energies [see Eqs. (28) and (29)], ${ }^{3}$ we obtain a cubic equation that relates the ratio between the virial $\left(R_{c}\right)$ to the turnaround outer radius $\left(R_{t}\right)$, the so-called collapse factor $\left(\lambda=R_{c} / R_{t}\right)$ :

$$
q_{2}(\gamma) n_{c} \lambda^{3}-\left(2+n_{t}\right) \lambda+2 q_{1}(\gamma)=0,
$$

where

$$
n_{c}=\frac{\Lambda\left(a_{c}\right)}{4 \pi G \rho_{m, t} \zeta}=n_{0}+\frac{2 \gamma a_{t}^{3(1-\gamma)}}{\zeta(1-\gamma)}\left[a_{c}^{-3(1-\gamma)}-1\right]
$$

and

$$
n_{t}=\frac{\Lambda\left(a_{t}\right)}{4 \pi G \rho_{m, t} \zeta}=n_{0}+\frac{2 \gamma a_{t}^{3(1-\gamma)}}{\zeta(1-\gamma)}\left[a_{t}^{-3(1-\gamma)}-1\right]
$$

with

$$
n_{0}=\frac{2 \Omega_{\Lambda} a_{t}^{3(1-\gamma)}}{\Omega_{m} \zeta} .
$$

Finally, solving the cubic Eq. (45), we calculate the collapse factor (see Appendix B). In the case of $\gamma=0\left(n_{c}=\right.$ $n_{t}=n_{0}$ ), the above expressions take the usual form of the $\Lambda$ cosmology (see [47,53]), as expected. Obviously, for the Einstein-de Sitter model $\left(\Omega_{m}=1, \gamma=0\right)$ we have $\Omega_{\Lambda}=0$ and all coefficients vanish $n_{c}=n_{t}=n_{0}=0$, so that Eq. (45) boils down to $\lambda=q_{1}(0)=1 / 2$; see Eq. (43).

In this framework, the density contrast at the virialization epoch is given by

$$
\Delta_{\mathrm{vir}}=\frac{\rho_{m s, c}}{\rho_{m, c}}=\frac{\zeta}{\lambda^{3}}\left(\frac{a_{c}}{a_{t}}\right)^{3},
$$

where $\rho_{m s, c}$ is the matter density in the virialized structure and $\rho_{m, c}$ is the background matter density at the same epoch. Following the notations of $[54,73,74]$, we again provide in Appendix C a fitting formula for $\Delta_{\text {vir }}$ (within a physical range of cosmological parameters $0 \leq \gamma \leq$ $0.01)$. Notice that the Einstein-de Sitter value for $\Delta_{\text {vir }}$ is precisely $18 \pi^{2}$, and was factorized in (C3). ${ }^{4}$

\footnotetext{
${ }^{3}$ In view of the fact that $U_{\Lambda}=-\Lambda(a) M R^{2} / 10$, the time dependence of the vacuum energy density seems to create a problem since the total energy of the bound system is not conserved. However, one can show that if the value of $|\gamma|$ is less that 0.01 then the problem of energy conservation does not really affect the virialization process and thus Eq. (42) remains a good approximation.

${ }^{4}$ This value ensues from the Einstein-de Sitter value of $\zeta=$ $(3 \pi / 4)^{2}$ multiplied by $2^{5}$. Indeed, the sphere contracts a factor of 2 from the turnaround point to virialization, and the background scale factor increases $a_{c} / a_{t}=2^{2 / 3}$, thus $\left(2 \times 2^{2 / 3}\right)^{3}=2^{5}$.
} 


\section{B. Clustered vacuum energy}

In this section we consider a scenario in which the vacuum energy density on the scale of galaxy clusters is clustered: $\rho_{\Lambda s}(R)=\Lambda_{s}(R) / 8 \pi G$. In such a scenario it could be possible, on nonlinear scales, to have an interaction between dark matter and dark energy with a different $\gamma$ than the background value. In the overdensity rest frame and in the homogeneous case (described before), the dark energy component flows progressively out of the overdensity $[58,62]$, and hence energy conservation cannot be applied (especially for large values of $\gamma$ 's) in order to determine the collapse factor $\lambda$ (along with the virial theorem). To simplify the inhomogeneous case formalism, we consider the extreme situation in which the vacuum energy fully clusters along with the dark matter, avoiding energy nonconservation which was examined in [58].

Within this framework, we also assume that the general functional form that describes the behavior of the vacuum energy density inside the spherical perturbation obeys a similar equation as that of Eq. (21):

$$
\Lambda_{s}(R)=\Lambda_{0}+8 \pi G \frac{\gamma_{s} \rho_{m s, t}}{1-\gamma_{s}}\left[\left(\frac{R}{R_{t}}\right)^{-3\left(1-\gamma_{s}\right)}-1\right]
$$

or else,

$$
\Lambda_{s}(y)=\Lambda_{0}+8 \pi G \frac{\gamma_{s} \zeta \rho_{m, t}}{1-\gamma_{s}}\left[y^{-3\left(1-\gamma_{s}\right)}-1\right]
$$

where the slope $\gamma_{s}$ is not necessarily equal to the background $\gamma$. It is worth noting that if we leave $\gamma_{s}$ to take also negative values then there is a critical radius $R_{\star}$, of the spherical overdensity, in which, for $R<R_{\star}$, the inhomogeneous vacuum energy density becomes negative $\Lambda_{s}<0$. Thus, the extra positive pressure $\left(P_{\Lambda_{s}}>0\right)$ inside the spherical perturbation implies compression (similar to gravity) rather than tension (while the opposite is true for $R>R_{\star}$ ). With the aid Eq. (51), this critical radius is given by

$$
R_{\star}=R_{t}\left[1-\frac{n_{0}\left(1-\gamma_{s}\right)}{2 \gamma_{s}}\right]^{-1 / 3\left(1-\gamma_{s}\right)}
$$

In the previous equation, we borrowed the definition of $n_{0}$ given in (48), where $\rho_{m s, t}=\rho_{m, t} \zeta=\rho_{m 0} a_{t}^{3(1-\gamma)} \zeta$ is used, in which $\gamma\left(\right.$ not $\left.\gamma_{s}\right)$ is involved because it refers to the evolution of the background matter density.

On the other hand, if $\gamma_{s} \geq 0$ then $\Lambda_{s}>0$ (or $P_{\Lambda_{s}}<0$ ) for all values of $0<y \leq 1$. In this paper we use 2 different versions of $\Lambda_{s}$, namely $\gamma_{s}=\gamma=0.002$ (hereafter $\Lambda_{\mathrm{RGC1}}$ ) and $\gamma_{s}=-0.002$ (hereafter $\Lambda_{\mathrm{RGC} 2}$ ). Of course, for the basic cosmological functions $\left[\Omega_{m}(a), E(a)\right.$, and $\left.D(a)\right]$ which enter in this vacuum pattern, we utilize the background $\gamma=0.002$.
Inserting Eq. (51) into Eq. (37), we obtain

$$
\ddot{y}=-\frac{H_{t}^{2} \Omega_{m, t}}{2}\left[\frac{\left(1-3 \gamma_{s}\right) \zeta}{\left(1-\gamma_{s}\right) y^{2-3 \gamma_{s}}}-2\left(r-\frac{\gamma_{s} \zeta}{1-\gamma_{s}}\right) y\right] .
$$

In contrast to the homogeneous case, the novelty of the current approach is that the above differential equation can be solved analytically. Indeed, due to the fact that the differential Eq. (53) is a function only of $y$ we can perform easily the integration

$$
\dot{y}^{2}=H_{t}^{2} \Omega_{m, t}[P(y, \zeta)+C],
$$

where $C$ is the integration constant and

$$
P(y, \zeta)=\frac{\zeta}{\left(1-\gamma_{s}\right) y^{1-3 \gamma_{s}}}+\left(r-\frac{\gamma_{s} \zeta}{1-\gamma_{s}}\right) y^{2} .
$$

Using now Eq. (40) we can provide the basic differential equation for the evolution of the overdensity perturbations

$$
\left(\frac{d y}{d x}\right)^{2}=\frac{P(y, \zeta)+C}{x^{-1+3 \gamma}+r x^{2} I(x)},
$$

where the boundary conditions, $(d y / d x)=0$ and $y=1$ at $x=1$, imply that $C=-P(1, \zeta)$. Therefore, the general integral equation which governs the behavior of the density contrast $\zeta$ at the turnaround epoch, for the RG vacuum models is

$$
\int_{0}^{1} \frac{d y}{\sqrt{P(y, \zeta)+C}}=\int_{0}^{1} \frac{d x}{\sqrt{x^{-1+3 \gamma}+r x^{2} I(x)}} .
$$

Of course, the usual $\Lambda \mathrm{CDM}$ cosmology is fully recovered from this model in the limit $\gamma=\gamma_{s}=0$.

Similarly, as in Sec. IVA, we again provide a useful fitting formula for $\zeta$ as well as for $\Delta_{\text {vir }}$, as a function of the cosmological parameters (for more details see Appendix C).

Now with the aid of Eq. (50), we can integrate Eq. (29) in order to derive the potential energy associated with the vacuum energy inside the spherical overdensity. In particular, we show here that $U_{\Lambda}$ can be written as a sum of three components that contribute to the local dynamics

$$
\begin{aligned}
U_{\Lambda}= & -\frac{M \Lambda_{0}}{10} R^{2}+4 \pi G \frac{\gamma_{s} M \rho_{m s, t}}{5\left(1-\gamma_{s}\right)} R^{2} \\
& -4 \pi G \frac{\gamma_{s} M \rho_{m s, t}}{\left(1-\gamma_{s}\right)\left(2+3 \gamma_{s}\right) R_{t}^{-3(1-\gamma)}} R^{-1+3 \gamma_{s},}
\end{aligned}
$$

where $\rho_{m s, t}=\zeta \rho_{m, t}$ (see Sec. IV). In this case, the algebraic equation which defines the collapse factor is found from the combination of equations (28), (42), and (58) as follows:

$$
\begin{aligned}
& q_{2}\left(\gamma_{s}\right)\left[n_{0}-f\left(\gamma_{s}\right)\right] \lambda^{3}-A\left(n_{0}, \gamma_{s}\right) \lambda+g\left(\gamma_{s}\right) \lambda^{3 \gamma_{s}} \\
& \quad+2 q_{1}\left(\gamma_{s}\right)=0,
\end{aligned}
$$

where $n_{0}$ is defined in Eq. (48), with 


$$
f\left(\gamma_{s}\right)=\frac{2 \gamma_{s}}{1-\gamma_{s}}, \quad g\left(\gamma_{s}\right)=\frac{10 \gamma_{s} q_{2}\left(\gamma_{s}\right)}{\left(1-\gamma_{s}\right)\left(2-3 \gamma_{s}\right)}
$$

and

$$
A\left(n_{0}, \gamma_{s}\right)=2+n_{0}-f\left(\gamma_{s}\right)+\frac{g\left(\gamma_{s}\right)}{q_{2}\left(\gamma_{s}\right)}
$$

Finally, solving Eq. (57) [or using Eq. (C1)] and (59), we can estimate the density contrast at virialization from Eq. (49).

\section{COMPARISON AMONG DIFFERENT TYPES OF VACUUM}

In this section, we investigate in more detail and compare the spherical collapse model, using different versions of the $\Lambda(t)$ model (homogeneous or clustered), with that of the traditional $\Lambda$ cosmology (see Appendix A). In Fig. 2 we present the density contrast at the turnaround point, $\zeta$, as a function of the turnaround redshift, $z_{t}$, for the constant vacuum $\Lambda$ (solid points), homogeneous vacuum $\Lambda_{\mathrm{RGH}}$ (solid line), clustered vacuum $\Lambda_{\mathrm{RGCl}}$ (dotted line), and clustered vacuum $\Lambda_{\mathrm{RGC} 2}$ (dashed line). It is obvious that the $\Lambda$ and $\Lambda_{\mathrm{RGH}}$ models are almost indistinguishable, while $\zeta$ appears to be somewhat lower in the inhomogeneous (clustered) case. Indeed, we find that $\zeta_{c l} / \zeta_{h} \approx \pi / 3$ at large redshifts.

Solving now Eqs. (45) and (59) we calculate the collapse factor and we find that it lies, in general, in the range $0.46 \leq \lambda \leq 0.52$, in agreement with previous studies $[25,53,56,58-60,63]$. In the upper panel of Fig. 3 we plot the deviation, $\left(1-\lambda / \lambda_{\Lambda}\right) \%$, of the collapse factors, $\lambda\left(z_{c}\right)$, for the current vacuum models with respect to the $\Lambda$ solution, $\lambda_{\Lambda}\left(z_{c}\right)$. It becomes evident that the size of the cosmic structures which are produced in the $\Lambda_{\mathrm{RGH}}$ model (solid line) is remarkably close to that predicted by the usual $\Lambda$ cosmology, and therefore the impact of the vacuum energy on the spherical collapse is very small in the homogeneous case. This was to be expected.

On the other hand, when considering the effect of the clustered vacuum energy, the largest positive deviation of the collapse factor occurs for the $\Lambda_{\mathrm{RGC} 2}$ model (dotted line), which implies that this model produces more bound systems than the concordance $\Lambda \mathrm{CDM}$ model. Therefore, within this vacuum pattern the corresponding cosmic structures should be located in larger density environments. The opposite situation holds for the $\Lambda_{\mathrm{RGCl}}$ (dashed line) model due to its negative deviations.

In Fig. 3 we plot the evolution of the density contrast at virialization. At very large redshifts, it tends to the Einstein-de Sitter value $\left(\Delta_{\text {vir }} \sim 18 \pi^{2}\right)$, as it should.

In Table I we list, for the case of a cluster forming at $z_{c} \simeq 0$ or at $z_{c} \simeq 1.6$, the following: (a) the cosmological models and the value of the turnaround redshift; and (b) the virial density $\Delta_{\text {vir }}$ at the collapse time, as well as the

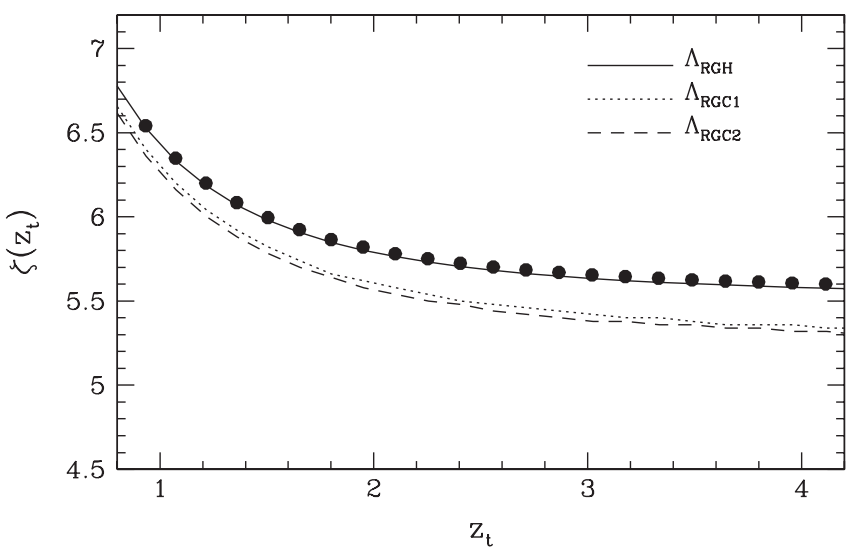

FIG. 2. The density contrast at the turnaround epoch, $\zeta$ as a function of the turnaround redshift. The lines represent the following cosmological models: (a) $\Lambda_{\mathrm{RGH}}$ (solid line), (b) $\Lambda_{\mathrm{RGC} 1}$ (dotted line, $\gamma_{s}=0.002$ ), and (c) $\Lambda_{\mathrm{RGC} 2}$ (dashed line, $\left.\gamma_{s}=-0.002\right)$. The points represent the concordance $\Lambda \mathrm{CDM}$ cosmology.

density excess of the matter density in the spherical overdensity, $\zeta$, at the turnaround time.

We also verify that the density contrast decreases with the formation (virialization) redshift $z_{c}$. The $\Delta_{\text {vir }}$

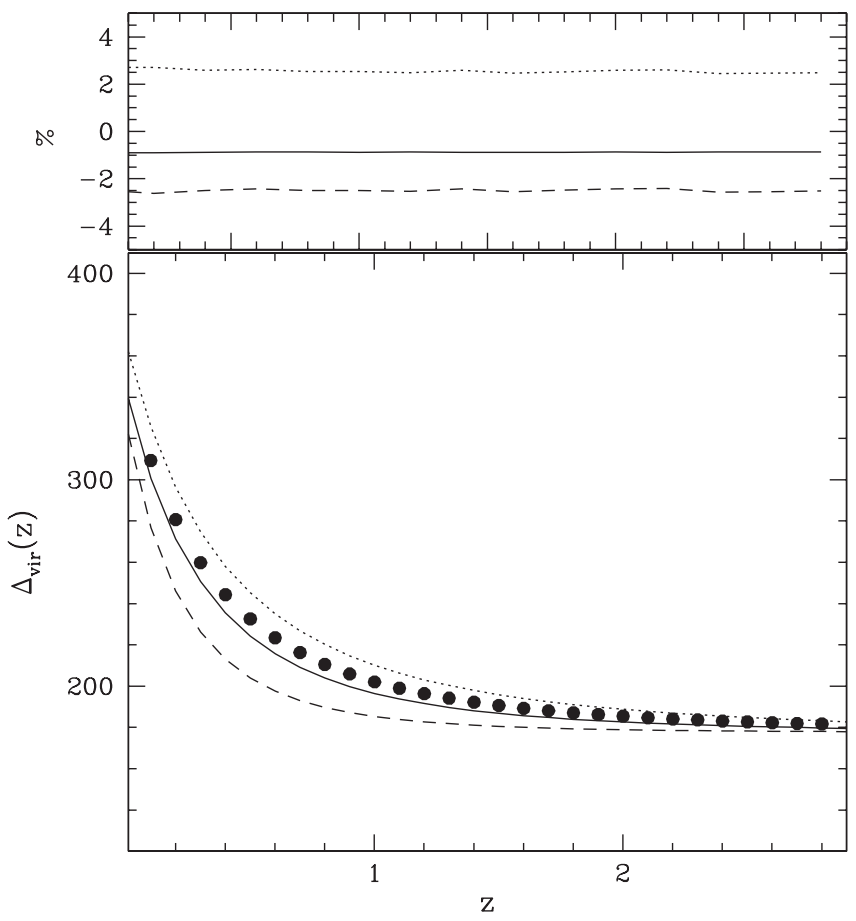

FIG. 3. Upper panel: The deviation $\left(1-\lambda / \lambda_{\Lambda}\right) \%$ of the collapse factor for various vacuum models with respect to the $\Lambda$ solution. Bottom panel: The density contrast at the virialization, $\Delta_{\text {vir }}$, as a function of redshift. The lines represent the following cosmological models: (a) $\Lambda_{\mathrm{RGH}}$ (solid line), (b) $\Lambda_{\mathrm{RGC} 1}$ (dotted line), and (c) $\Lambda_{\mathrm{RGC} 2}$ (dashed line). The points represent the traditional $\Lambda$ cosmology. 
differences among the different vacuum models enter through $\Omega_{m}(z)$ [see Eq. (C3)] as well as through the assumption about the behavior of the vacuum inside the spherical overdensity (homogeneous or not). This feature points to the fact that perhaps the density contrast at virialization can be used as an effective cosmological tool.

Although, we will investigate in detail such a possibility in a forthcoming paper, we present here an idea of how to use observational data to estimate $\Delta_{\text {vir }}$. Using existing catalogs of clusters of galaxies, one should select those clusters which appear to have a quasispherical projected shape, ${ }^{5}$ which is the expected shape of a virialized cosmic structure, and then derive their virial radius and mass. One can then easily calculate the observational value of $\Delta_{\text {vir }}$ of a cluster at a redshift $z$, from

$$
\Delta_{\mathrm{vir}}=\frac{3 M_{\mathrm{vir}}}{4 \pi \rho_{\mathrm{crit}, 0} \Omega_{m, 0}(1+z)^{3} r_{\mathrm{vir}}^{3}}
$$

and compare it with the model expectations. Now, the cluster virial radius can be calculated from the projected separations of the $N_{m}$ galaxy members according to (e.g., [75])

$$
r_{\mathrm{vir}}=\frac{\pi}{2} \frac{N_{m}\left(N_{m}-1\right)}{\sum_{i=1}^{N_{m}-1} \sum_{j=i+1}^{N_{m}}\left[d_{L} \tan \left(\delta \theta_{i j}\right)\right]^{-1}},
$$

where $d_{L}$ is the luminosity distance of the group and $\delta \theta_{i j}$ is the angular $(i, j)$-pair separation. Using the observed cluster velocity dispersion, $\sigma_{v}$, and $r_{\text {vir }}$ one can estimate the cluster's virial mass using the virial theorem, according to

$$
M_{\mathrm{vir}}=\frac{3 \sigma_{v}^{2} r_{\mathrm{vir}}}{G}+\frac{\Lambda r_{\mathrm{vir}}^{3}}{5 G} .
$$

The second $\Lambda$-based term is negligible, $\sim 4.7 \times$ $10^{11} \Omega_{\Lambda} r_{\text {vir }}^{3} M_{\odot}$, and therefore it does not affect significantly the mass estimates of clusters of galaxies. Of course, this approach is of a statistical nature, since there are various observational systematics that enter in the individual cluster determination of $\Delta_{\text {vir }}$, as well as cosmic variance, which however can be minimized if one averages over a suitable and relatively large sample of clusters at each redshift interval.

We have applied this methodology to the 2MASS High Density Contrast group catalog [76], which is a low- $z$ catalog based on the $2 \mu \mathrm{m}$ infrared whole-sky survey and which was constructed by a friends-of-friends algorithm (e.g. [77]) such that the groups correspond to an overdensity $\delta \rho / \rho \geq 80$. This catalog was carefully constructed, with respect to other catalogs, and it is less prone to projection, interloper contamination, and contamination by the large-scale structures from which galaxies are ac-

\footnotetext{
${ }^{5}$ Not all apparently spherical clusters are truly spherical since elongated clusters with their major axis oriented at small angles along the line of sight will appear spherical in projection, a fact which is a further source of noise.
}

TABLE I. Numerical results: The 1st column indicates the vacuum model used. Between columns two and four, we present the main properties of the spherical collapse model $\left[\Delta_{\text {vir }}\left(z_{c}\right), \zeta\left(z_{t}\right)\right]$, assuming that galaxy clusters have collapsed prior to the present time $z_{c} \simeq 0\left(z_{t} \simeq 0.75\right)$. In columns four and five, we give the same quantities but considering that clusters have formed (collapsed) prior to the epoch of $z_{c} \simeq 1.6\left(z_{t} \simeq 3.2\right.$ ), in which the most distant cluster has been found.

\begin{tabular}{lcccc}
\hline \hline Model & $\Delta_{\text {vir }}(0)$ & $\zeta(0.75)$ & $\Delta_{\text {vir }}(1.6)$ & $\zeta(3.2)$ \\
\hline$\Lambda$ & 348.6 & 6.80 & 190.0 & 5.64 \\
$\Lambda_{\text {RGH }}$ & 339.0 & 6.79 & 184.3 & 5.62 \\
$\Lambda_{\text {RGC1 }}$ & 317.6 & 6.66 & 168.6 & 5.40 \\
$\Lambda_{\text {RGC2 }}$ & 368.8 & 6.62 & 195.2 & 5.38 \\
\hline \hline
\end{tabular}

creted to the groups (see [78] for a relevant discussion). We selected only those groups with projected axial ratio $>0.8$ and with at least 16 galaxy members (in order to have a relatively accurate determination of their shape, velocity dispersion, and thus $M_{\text {vir }}$ ) and we are thus left with 7 clusters at $\langle z\rangle \simeq 0.015$. We clip the lower and higher $\Delta_{\text {vir }}$ outliers, since we do expect systematic effects to be present, and we derive a mean value of $\left\langle\Delta_{\text {vir }}\right\rangle=348$ and a standard deviation of the distribution of \pm 73 (if we use $N_{m}>20$ we are left with 6 clusters with $\left\langle\Delta_{\text {vir }}\right\rangle=329 \pm$ 69). Although, we have derived these $\Delta_{\text {vir }}$ values using the concordance $\Lambda \mathrm{CDM}$ cosmological model to estimate $d_{L}$, there would be no appreciable difference had we used any of the other models, presented in Sec. II A (because of the very small value of $\gamma$ and of the very low redshift of the sample). Inspecting Table I it is evident that the previously derived observational values are in good agreement with the theoretical expectations although with the present level of uncertainty we cannot distinguish among the models. However, in the case of the clustered vacuum energy model

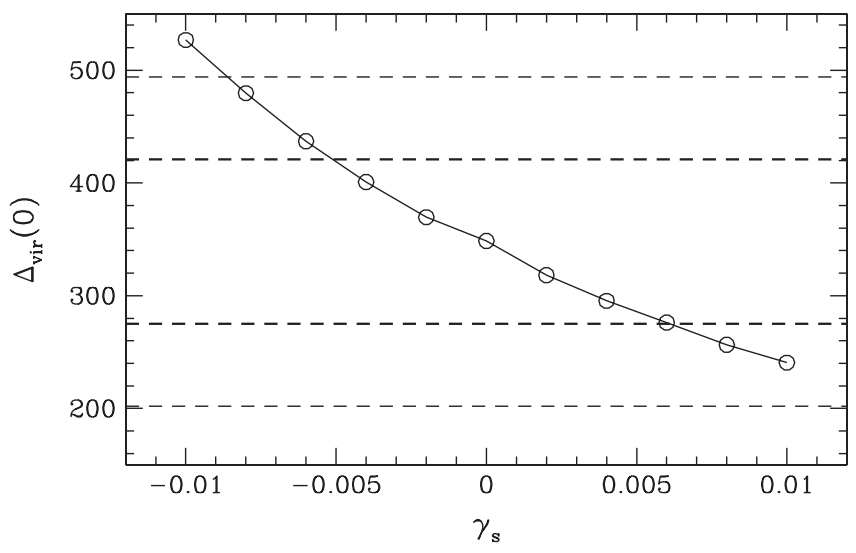

FIG. 4. The present time virial density for the clustered vacuum energy model as a function of $\gamma_{s}$ (open points). The inner and outer dashed lines correspond to the 1 and $2 \sigma$, observationally determined, virial density limits, respectively $(202 \leq$ $\Delta_{\text {vir }} \leq 494$ ), based on a subsample of the 2MASS High Density Contrast group catalog. 
we can put some limits on the value of $\gamma_{s}$ even with the present level of accuracy. As an example, we compare in Fig. 4 , the predicted virial density, $\Delta_{\text {vir }}$, of the model at the present time (open points), with the observationally derived $2 \sigma$ range of values, based on the previously discussed subsample of the 2MASS High Density Contrast group catalog, and find a consistency for $-0.009 \lesssim \gamma_{s} \lesssim$ 0.012. In the future we plan to further investigate the model predictions, using a larger number of clusters spanning also a range of different redshifts, in an attempt to put stringent constraints on the value of $\gamma_{s}$.

\section{CONCLUSIONS}

In this paper we have studied analytically and numerically the spherical collapse model in the case of a time varying vacuum, with $\Lambda(H)=n_{0}+n_{2} H^{2}$, for a spatially flat FLRW geometry. We find that the amplitude and the shape of the virial density contrast is affected by the considered status of the vacuum energy model (homogeneous or clustered). We verify that in the case where the distribution of the vacuum energy is clustered the structures produced are more concentrated (under specific conditions) with respect to the homogeneous dark energy case. Finally, by comparing the predicted virial density contrast at the present epoch with a preliminary analysis of a suitable subsample of the 2MASS High Density Contrast group catalog (at a mean redshift of $\langle z\rangle \simeq 0.015$ ), we find that the inhomogeneous vacuum energy models can be accommodated, at a $2 \sigma$ level, if the vacuum clustering parameter is within the range $-0.009 \lessgtr \gamma_{s} \lesssim 0.012$. The latter result points to the direction that perhaps the $\Delta_{\text {vir }}$ parameter, once estimated accurately from observations, could be used in order to determine the internal physical properties of the vacuum energy.

\section{ACKNOWLEDGMENTS}

J.S. has been supported in part by MEC and FEDER under Project No. FPA2007-66665, by the Spanish Consolider-Ingenio 2010 Program CPAN CSD200700042, and by DIUE/CUR Generalitat de Catalunya under Project No. 2009SGR502. M. P. acknowledges funding by Mexican CONACyT Grant No. 2005-49878.

\section{APPENDIX A: THE CONCORDANCE $\Lambda$ COSMOLOGY}

In this Appendix we would like to give the reader the opportunity to appreciate the fact that the $\Lambda_{\mathrm{RG}}$ model can be viewed as an extension of the concordance $\Lambda$ cosmology. In particular, the basic cosmological equations in the $\Lambda_{\mathrm{RG}}$ model reduce to those of the $\Lambda$ cosmology for $\gamma=0$. Below, we present the main quantities of the $\Lambda$ cosmology:

(i) Global dynamics: The basic cosmological equations (see Sec. II A) take the following forms:

$$
\begin{gathered}
H(t)=\sqrt{\Omega_{\Lambda}} H_{0} \operatorname{coth}\left[\frac{3 H_{0} \sqrt{\Omega_{\Lambda}}}{2} t\right], \\
a(t)=\left(\frac{\Omega_{m}}{\Omega_{\Lambda}}\right)^{1 / 3} \sinh ^{2 / 3}\left(\frac{3 H_{0} \sqrt{\Omega_{\Lambda}}}{2} t\right),
\end{gathered}
$$

and

$$
E^{2}(a)=\frac{H^{2}(a)}{H_{0}^{2}}=\Omega_{\Lambda}+\Omega_{m} a^{-3} .
$$

(ii) The spherical model: The basic set of equations here is

$$
\begin{aligned}
& \dot{x}^{2}=H_{t}^{2} \Omega_{m, t}\left[x^{-1}+r x^{2} I(x)\right], \\
& \ddot{y}=-\frac{H_{t}^{2} \Omega_{m, t}}{2}\left(\frac{\zeta}{y^{2}}-2 r y I(x)\right),
\end{aligned}
$$

where $I(x) \equiv 1$ and

$$
r=\frac{\Omega_{\Lambda, t}}{\Omega_{m, t}}=r_{0} a_{t}^{3},
$$

where $r_{0}=\Omega_{\Lambda} / \Omega_{m}$. Therefore, the general integral equation which governs the behavior of the density contrast $\zeta$ at the turnaround epoch is

$$
\int_{0}^{1} \frac{d y}{\sqrt{P(y, \zeta)-P(1, \zeta)}}=\frac{\ln (\sqrt{1+r}+\sqrt{r})^{2 / 3}}{\sqrt{r}},
$$

where

$$
P(y, \zeta)=\frac{\zeta}{y}+r y^{2}
$$

Note that the time needed for a spherical shell to collapse is twice the turnaround time, $t_{f} \simeq 2 t_{t}$. This implies that

$$
\sinh ^{-1}\left(\sqrt{r_{0} a_{c}^{3}}\right) \simeq 2 \sinh ^{-1}\left(\sqrt{r_{0} a_{t}^{3}}\right) .
$$

(iii) Virial theorem: The virial theorem becomes

$$
2 T+U_{G}-2 U_{\Lambda}=0 .
$$

Using now also the energy conservation at the turnaround and at the virial time we derive the following relations:

$$
\begin{gathered}
\frac{1}{2} U_{G, c}+2 U_{\Lambda, c}=U_{G, t}+U_{\Lambda, t}, \\
2 n_{0} \lambda^{3}-\left(2+n_{0}\right) \lambda+1=0,
\end{gathered}
$$

where $\lambda=R_{c} / R_{t}$ is the collapse factor and 


$$
n_{0}=\frac{2 \Omega_{\Lambda} a_{t}^{3}}{\Omega_{m} \zeta} .
$$

\section{APPENDIX B: ROOTS OF A CUBIC POLYNOMIAL}

We remind the reader of some basic elements of algebra pertinent to our analysis. Given a cubic equation $\lambda^{3}+$ $a_{1} \lambda^{2}+a_{2} \lambda+a_{3}=0$, let $D$ be the discriminant:

$$
\mathcal{D}=a_{1}^{2} a_{2}^{2}-4 a_{2}^{3}-4 a_{1}^{3} a_{3}-27 a_{3}^{2}+18 a_{1} a_{2} a_{3}
$$

and

$$
x_{1}=-a_{1}^{3}+\frac{9}{2} a_{1} a_{2}-\frac{27}{2} a_{3}, \quad x_{2}=-\frac{3 \sqrt{3 \mathcal{D}}}{2} .
$$

If $\mathcal{D}>0$, all roots are real (irreducible case). In that case $\lambda_{1}, \lambda_{2}$, and $\lambda_{3}$ can be written:

$$
\begin{aligned}
& \lambda_{\mu}=-\frac{a_{1}}{3}-\frac{2 r^{1 / 3}}{3} \cos \left[\frac{\theta-(\mu-1) \pi}{3}\right], \\
& \mu=1,2,3,
\end{aligned}
$$

where $r=\sqrt{x_{1}^{2}+x_{2}^{2}}$ and $\theta=\cos ^{-1}\left(x_{1} / r\right)$.

Now, we are ready to derive analytically the exact roots of the basic cubic equation (45) having polynomial parameters $a_{1}=0, \quad a_{2}=-\left(2+n_{t}\right) / q_{2} n_{c}, \quad$ and $a_{3}=$ $2 q_{1} / q_{2} n_{c}$. Then the discriminant becomes

$$
\mathcal{D}\left(n_{t}, n_{c}\right)=4 \frac{\left(2+n_{t}\right)^{3}-27 q_{1}^{2} q_{2} n_{c}}{q_{2}^{3} n_{c}^{3}} .
$$

Of course, in order to obtain physically acceptable solutions we need to take $n_{t}, n_{c}>0$, which gives $\mathcal{D}\left(n_{t}, n_{c}\right)>$ 0 . Therefore, all roots of the cubic equation are real (irreducible case) but one of them $0 \leq \lambda_{3} \leq 1$ corresponds to expanding shells. It is obvious that for $n_{t}, n_{c} \rightarrow 0$, the

above solution tends to the Einstein-de Sitter case $\left(\lambda_{3} \rightarrow\right.$ $0.50)$, as it should.

\section{APPENDIX C: FITTING FORMULAS}

We provide here accurate fitting formulas for the density contrast at the turnaround redshift and at the epoch of virialization, and which do not have a simple fully analytical form. These are

$$
\zeta \simeq\left(\frac{3 \pi}{4} \sqrt{1+A_{s}}\right)^{2} \Omega_{m, t}^{-\omega_{1}+\omega_{2} \Omega_{m, t}-\omega_{3} w\left(a_{t}\right)},
$$

where

$$
w(a)=-1-\frac{\gamma a^{3 \gamma}}{a^{3 \gamma}+\tilde{r}_{0}},
$$

and

$$
\Delta_{\text {vir }}(a) \simeq 18 \pi^{2}\left[1+\epsilon \Theta^{b}(a)\right],
$$

where $\Theta(a)=\Omega_{m}^{-1}(a)-1$.

(i) Homogeneous vacuum: In this case we have $A_{s}=0$, $\left(\omega_{1}, \omega_{2}, \omega_{3}\right)=(0.79,0.26,0.06)$ and

$$
\epsilon=0.40-25 \gamma+500 \gamma^{2}, \quad b=0.94+50 \gamma .
$$

(ii) Clustered vacuum: Here we find

$$
A_{s}= \begin{cases}-24.25 \gamma_{s}+2125 \gamma_{s}^{2} & 0 \leq \gamma_{s} \leq 0.01 \\ 29.75 \gamma_{s}+2375 \gamma_{s}^{2} & -0.01 \leq \gamma_{s}<0,\end{cases}
$$

$$
\left(\omega_{1}, \omega_{2}, \omega_{3}\right)= \begin{cases}(0.86,0.36,0) & 0 \leq \gamma_{s} \leq 0.01, \\ (0.74,0.16,0) & -0.01 \leq \gamma_{s}<0,\end{cases}
$$

$$
b= \begin{cases}0.94+145 \gamma_{s}+4.75 \times 10^{4} \gamma_{s}^{2} & -0.002 \leq \gamma_{s} \leq 0.01, \\ 0.94+55 \gamma_{s} & -0.01 \leq \gamma_{s}<-0.002,\end{cases}
$$

and

$$
\epsilon= \begin{cases}0.40-65 \gamma_{s}-1.25 \times 10^{4} \gamma_{s}^{2} & -0.002 \leq \gamma_{s} \leq 0.01 \\ 0.31-86.25 \gamma_{s} & -0.01 \leq \gamma_{s}<-0.002\end{cases}
$$

[1] M. Tegmark et al., Astrophys. J. 606, 702 (2004).

[2] D. N. Spergel et al., Astrophys. J. Suppl. Ser. 170, 377 (2007).

[3] T. M. Davis et al., Astrophys. J. 666, 716 (2007).
[4] M. Kowalski et al., Astrophys. J. 686, 749 (2008).

[5] M. Hicken et al., Astrophys. J. 700, 1097 (2009).

[6] E. Komatsu et al., Astrophys. J. Suppl. Ser. 180, 330 (2009); arXiv:1001.4538. 
[7] S. Weinberg, Rev. Mod. Phys. 61, 1 (1989).

[8] P. J. Peebles and B. Ratra, Rev. Mod. Phys. 75, 559 (2003).

[9] T. Padmanabhan, Phys. Rep. 380, 235 (2003).

[10] L. Perivolaropoulos, arXiv.0811.4684.

[11] P. J. Steinhardt, Critical Problems in Physics, edited by V.L. Fitch, D.R. Marlow, and M.A.E. Dementi (Princeton University Press, Princeton, NJ, 1997); Phil. Trans. R. Soc. A 361, 2497 (2003).

[12] C.A. Egan and C.H. Lineweaver, Phys. Rev. D 78, 083528 (2008).

[13] I. L. Shapiro and J. Solà, Phys. Lett. B 475, 236 (2000).

[14] I. L. Shapiro and J. Solà, J. High Energy Phys. 02 (2002) 006.

[15] A. Bonanno and M. Reuter, Phys. Rev. D 62, 043008 (2000); M. Reuter and H. Weyer, Phys. Rev. D 70, 124028 (2004).

[16] A. Babić, B. Guberina, R. Horvat, and H. Štefančić, Phys. Rev. D 65, 085002 (2002); B. Guberina, R. Horvat, and H. Štefančić, Phys. Rev. D 67, 083001 (2003); A. Babić, B. Guberina, R. Horvat, and H. Štefančić, Phys. Rev. D 71, 124041 (2005).

[17] J. Grande, J. Solà, and H. Štefančić, J. Cosmol. Astropart. Phys. 08 (2006) 011; Phys. Lett. B 645, 235 (2007).

[18] S. Basilakos, Astron. Astrophys. 508, 575 (2009).

[19] A. D. Dolgov, The Very Early Universe, edited by G. Gibbons, S. W. Hawking, and S. T. Tiklos (Cambridge University Press, Cambridge, England, 1982).

[20] B. Ratra and P. J.E. Peebles, Phys. Rev. D 37, 3406 (1988); R. D. Peccei, J. Solà, and C. Wetterich, Phys. Lett. B 195, 183 (1987); C. Wetterich, Nucl. Phys. B302, 668 (1988); M.S. Turner and M. White, Phys. Rev. D 56, R4439 (1997); R. R. Caldwell, R. Dave, and P. J. Steinhardt, Phys. Rev. Lett. 80, 1582 (1998).

[21] H. K. Jassal, J. S. Bagla, and T. Padmanabhan, Phys. Rev. D 72, 103503 (2005); Mon. Not. R. Astron. Soc. Lett. 356, L11 (2004).

[22] L. Samushia and B. Ratra, Astrophys. J. 650, L5 (2006); 680, L1 (2008).

[23] J. Q. Xia, H. Li, G. B. Zhao, and X. Zhang, Phys. Rev. D 78, 083524 (2008); G. B. Zhao, J. Q. Xia, B. Feng, and X. Zhang, Int. J. Mod. Phys. D 16, 1229 (2007); J. Q. Xia, G. B. Zhao, B. Feng, H. Li, and X. Zhang, Phys. Rev. D 73, 063521 (2006).

[24] J. Simon, L. Verde, and R. Jiménez, Phys. Rev. D 71, 123001 (2005).

[25] D. F. Mota and C. van de Bruck, Astron. Astrophys. 421, 71 (2004).

[26] N. J. Nunes and D. F. Mota, Mon. Not. R. Astron. Soc. 368, 751 (2006).

[27] M. Ozer and O. Taha, Nucl. Phys. B287, 776 (1987); O. K. Freese et al., Nucl. Phys. B 287, 797 (1987); O. Bertolami, Nuovo Cimento B 93, 36 (1986).

[28] O. Bertolami and P.J. Martins, Phys. Rev. D 61, 064007 (2000); J.S. Alcaniz and J. M.F. Maia, Phys. Rev. D 67, 043502 (2003); R. Opher and A. Pelinson, Phys. Rev. D 70, 063529 (2004); J. D. Barrow and T. Clifton, Phys. Rev. D 73, 103520 (2006); A.E. Montenegro, Jr. and S. Carneiro, Classical Quantum Gravity 24, 313 (2007).
[29] J. C. Carvalho, J. A. S. Lima, and I. Waga, Phys. Rev. D 46, 2404 (1992); I. Waga, Astrophys. J. 414, 436 (1993); J. Salim and I. Waga, Classical Quantum Gravity 10, 1767 (1993); A. I. Arbab, Gen. Relativ. Gravit. 29, 61 (1997).

[30] For a review, see J. M. Overduin and F. I. Cooperstock, Phys. Rev. D 58, 043506 (1998).

[31] L.E. Parker and D. J. Toms, Quantum Field Theory in Curved Spacetime, Quantized Fields and Gravity (Cambridge University Press, Cambridge, England, 2009).

[32] I. L. Shapiro and J. Solà, Phys. Lett. B 682, 105 (2009); see also the reviews I. L. Shapiro and J. Solà, J. Phys. A 40, 6583 (2007); arXiv:0808.0315 on the QFT of the cosmological term, and references therein.

[33] J. Grande, A. Pelinson, and J. Solà, Phys. Rev. D 79, 043006 (2009); J. Grande, R. Opher, A. Pelinson, and J. Solà, J. Cosmol. Astropart. Phys. 12 (2007) 007; J.C. Fabris, I. L. Shapiro, and J. Solà, J. Cosmol. Astropart. Phys. 02 (2007) 016.

[34] J. Grande, J. Solà, J. C. Fabris, and I. L. Shapiro, Classical Quantum Gravity 27, 105004 (2010).

[35] S. Basilakos, M. Plionis, and S. Solà, Phys. Rev. D 80, 083511 (2009).

[36] B. L. Nelson and P. Panangaden, Phys. Rev. D 25, 1019 (1982); E. S. Fradkin and A. A. Tseytlin, Nucl. Phys. B201, 469 (1982); S. L. Adler, Rev. Mod. Phys. 54, 729 (1982).

[37] D. J. Toms, Phys. Lett. 126B, 37 (1983); L. Parker and D. J. Toms, Phys. Rev. D 32, 1409 (1985); I. L. Buchbinder, Theor. Fortsch. Phys. 34, 605 (1986).

[38] I. L. Shapiro, J. Solà, C. España-Bonet, and P. RuizLapuente, Phys. Lett. B 574, 149 (2003); C. EspañaBonet, P. Ruiz-Lapuente, I. L. Shapiro, and J. Solà, J. Cosmol. Astropart. Phys. 02 (2004) 006; I. L. Shapiro and J. Solà, Nucl. Phys. B, Proc. Suppl. 127, 71 (2004).

[39] I. L. Shapiro, J. Solà, and H. Štefančić, J. Cosmol. Astropart. Phys. 01 (2005) 012.

[40] J. Solà and H. Štefančić, Phys. Lett. B 624, 147 (2005); Mod. Phys. Lett. A 21, 479 (2006); J. Phys. A 39, 6753 (2006).

[41] J. Solà, J. Phys. A 41, 164066 (2008).

[42] F. Bauer, Classical Quantum Gravity 22, 3533 (2005); Ph.D. thesis, Technische Universitat Munchen, 2006, arXiv:hep-th/0610178.

[43] F. Bauer, J. Solà, and H. Štefančić, Phys. Lett. B 678, 427 (2009).

[44] B. F. L. Ward, Mod. Phys. Lett. A 25, 607 (2010); Int. J. Mod. Phys. D 17, 627 (2008); Mod. Phys. Lett. A 23, 3299 (2008).

[45] J. E. Gunn and J. R. Gott, Astrophys. J. 176, 1 (1972).

[46] P.J.E. Peebles, Principles of Physical Cosmology (Princeton University Press, Princeton, NJ, 1993).

[47] O. Lahav, P. B. Lilje, J. R. Primack, and M. J. Rees, Mon. Not. R. Astron. Soc. 251, 128 (1991) [http://adsabs .harvard.edu/abs/1991MNRAS.251..128L].

[48] L. Wang and J.P. Steinhardt, Astrophys. J. 508, 483 (1998).

[49] I. T. Iliev and P. R. Shapiro, Mon. Not. R. Astron. Soc. 325, 468 (2001).

[50] E. L. Lokas, Acta Phys. Pol. B 32, 3643 (2001) [arXiv: astro-ph/0112031]. 
[51] R. A. Battye and J. Weller, Phys. Rev. D 68, 083506 (2003).

[52] R. Mainini, A.W. Maccio, S. A. Bonometto, and A. Klypin, Astrophys. J. 599, 24 (2003).

[53] S. Basilakos, Astrophys. J. 590, 636 (2003).

[54] N. N. Weinberg and M. Kamionkowski, Astrophys. J. 341, 251 (2003).

[55] M. Manera and D. F. Mota, Mon. Not. R. Astron. Soc. 371, 1373 (2006).

[56] C. Horellou and J. Berge, Mon. Not. R. Astron. Soc. 360, 1393 (2005).

[57] Ding-fang Zeng and Yi-hong Gao, arXiv:astro-ph/ 0505164.

[58] I. Maor and O. Lahav, J. Cosmol. Astropart. Phys. 07 (2005) 003.

[59] W. J. Percival, Astron. Astrophys. 443, 819 (2005).

[60] P. Wang, Astrophys. J. 640, 18 (2006).

[61] M. Bartelmann, M. Doran, and C. Wetterich, Astron. Astrophys. 454, 27 (2006); F. Pace, J. C. Waizmann, and M. Bartelman, arXiv:1005.0233 [Mon. Not. R. Astron. Soc. (to be published)].

[62] D. F. Mota, J. Cosmol. Astropart. Phys. 09 (2008) 006.

[63] S. Basilakos and N. Voglis, Mon. Not. R. Astron. Soc. 374, 269 (2007); S. Basilakos, J. C. Sanchez, and L. Perivolaropoulos, Phys. Rev. D 80, 043530 (2009).

[64] B. M. Schafer and K. Koyama, Mon. Not. R. Astron. Soc. 385, 411 (2008).

[65] S. Lee and Kin-Wang Ng, arXiv:0910.0126.

[66] L.R. Abramo, R.C. Batista, L. Liberato, and R. Rosenfeld, J. Cosmol. Astropart. Phys. 11 (2007) 012; Phys. Rev. D 79, 023516 (2009); Ph. Brax, R. Rosenfeld, and D. A. Steer, J. Cosmol. Astropart. Phys. 08 (2010) 033.

[67] D. C. Rodrigues, P.S. Letelier, and I. L. Shapiro, J. Cosmol. Astropart. Phys. 04 (2010) 020

[68] M. Bronstein, Phys. Z. Sowjetunion 3, 73 (1933).
[69] M. Chevallier and D. Polarski, Int. J. Mod. Phys. D 10, 213 (2001); E. V. Linder, Phys. Rev. Lett. 90, 091301 (2003).

[70] L. Amendola, Phys. Rev. D 62, 043511 (2000); W. Zimdahl, D. Pavón, and L. P. Chimento, Phys. Lett. B 521, 133 (2001); L. Amendola, C. Quercellini, D. Tocchini-Valentini, and A. Pasqui, Astrophys. J. 583, L53 (2003); G. Mangano, G. Miele, and V. Pettorino, Mod. Phys. Lett. A 18, 831 (2003); R. G. Cai and A. Wang, J. Cosmol. Astropart. Phys. 03 (2005) 002; J. B. Binder and G. M. Kremer, Gen. Relativ. Gravit. 38, 857 (2006); S. Das, P. S. Corasaniti, and J. Khoury, Phys. Rev. D 73, 083509 (2006); G. Huey and B. D. Wandelt, Phys. Rev. D 74, 023519 (2006); R. Mainini and S. Bonometto, Phys. Rev. D 74, 043504 (2006); B. Wang, C-Y. Lin, and Abdalla, Phys. Lett. B 637, 357 (2006); G. M. Kremer, Gen. Relativ. Gravit. 39, 965 (2007); A. W. Brookfield, C. van de Bruck, and L. M. H. Hall, Phys. Rev. D 77, 043006 (2008); G. Olivares, F. Atrio-Barandela, and D. Pavón, Phys. Rev. D 77, 063513 (2008); J.-H. He and B. Wang, J. Cosmol. Astropart. Phys. 06 (2008) 010.

[71] T. Padmanabhan, Structure Formation in the Universe (Cambridge University Press, Cambridge, England, 1993).

[72] C. Papovich et al., Astrophys. J. 716, 1503 (2010).

[73] T. Kitayama and Y. Suto, Astrophys. J. 469, 480 (1996).

[74] D. J. Shaw and D. F. Mota, Astrophys. J. Suppl. Ser. 174, 277 (2008).

[75] M.E. Merchán and A. Zandivarez, Astrophys. J. 630, 759 (2005).

[76] A. C. Crook, J. P. Huchra, N. Martimbeau, K. L. Masters, T. Jarrett, and L. M. Macri, Astrophys. J. 655, 790 (2007).

[77] J.P. Huchra and M. J. Geller, Astrophys. J. 257, 423 (1982).

[78] H. Tovmassian and M. Plionis, Astrophys. J. 696, 1441 (2009). 